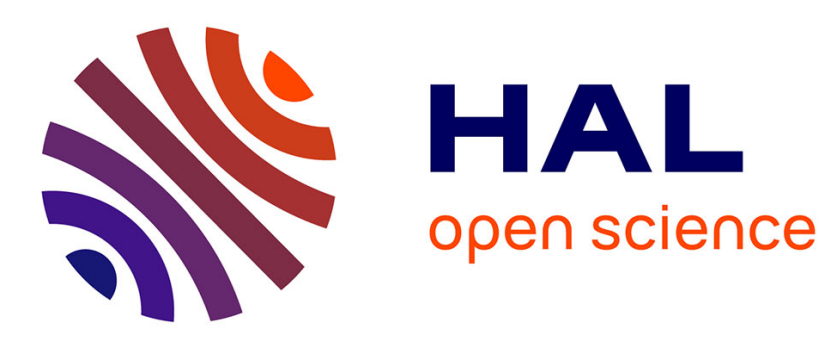

\title{
Crambescidin Acid from the French Polynesian Monanchora n. sp. Marine Sponge
}

\author{
A. El-Demerdash, S. Petek, C. Debitus, A. Al-Mourabit
}

\section{To cite this version:}

A. El-Demerdash, S. Petek, C. Debitus, A. Al-Mourabit. Crambescidin Acid from the French Polynesian Monanchora n. sp. Marine Sponge. Chemistry of Natural Compounds, 2020, 56 (6), pp.1180 - 1182. 10.1007/s10600-020-03262-1 . hal-03047904

\section{HAL Id: hal-03047904 https://hal.science/hal-03047904}

Submitted on 1 Mar 2021

HAL is a multi-disciplinary open access archive for the deposit and dissemination of scientific research documents, whether they are published or not. The documents may come from teaching and research institutions in France or abroad, or from public or private research centers.
L'archive ouverte pluridisciplinaire HAL, est destinée au dépôt et à la diffusion de documents scientifiques de niveau recherche, publiés ou non, émanant des établissements d'enseignement et de recherche français ou étrangers, des laboratoires publics ou privés. 


\title{
CRAMBESCIDIN ACID FROM THE FRENCH POLYNESIAN Monanchora n. sp. MARINE SPONGE
}

\author{
A. El-Demerdash, ${ }^{1,2 *}$ S. Petek, ${ }^{3,4}$ C. Debitus, ${ }^{3,4}$ \\ and A. Al-Mourabit 1
}

Marine natural products continue to be rich reservoirs for pharmacological lead compounds [1, 2]. Marine sponges are a distinct multicellular phylum and represent the biggest class of marine invertebrates with approximately 8,900 registered species, though 15,000 species are distributed over seas and oceans [3-5]. Monanchora marine sponges are well recognized for producing a high number of structurally divergent bioactive secondary metabolites [6], including acyclic and polycyclic guanidine alkaloids [7-9], steroids [10], terpenoids [11, 12], and long chain fatty acids [13]. These compounds display a myriad of biological potentialities as antimalarial [14], anticancer [15], anti-infective [16], and antiviral [17]. As a part of our ongoing program on bioactive natural products from marine sponges [18-21], we report herein the isolation and identification of five natural products $\mathbf{1 - 5}$ for the first time from the $n$-BuOH fraction of Monanchora $\mathrm{n}$. sp. sponge.

Chemical investigation of the French Polynesia Monanchora sp. marine sponge led to the isolation and identification of one pentacyclic guanidine alkaloid crambescidin acid (1) along with four simple nitrogenous compounds, including one nucleoside thymidine (2) and three amino acid derivatives, namely phenylethylamine (3), tryptophan (4). and phenylalanine (5).

Compound 1 (11.5 mg) was purified as a viscous pale yellow oily material. It has the molecular formula $\mathrm{C}_{22} \mathrm{H}_{33} \mathrm{~N}_{3} \mathrm{O}_{4}$ as established from its positive HR-ESI-MS $m / z$ 404.2461 [M $+\mathrm{H}]^{+}$(calcd 404.2549), indicating eight degrees of unsaturation. A preliminary inspection of its ${ }^{1} \mathrm{H}$ NMR recorded in $\mathrm{MeOH}-\mathrm{d}_{4}$ showed characteristic signals with similar chemical shifts to those reported previously for crambescidine pentacyclic guanidine alkaloids, confirming the presence of a pentacyclic guanidinic core (vessel), which accounts for five degrees of unsaturation. A detailed analysis of the ${ }^{13} \mathrm{C}$ NMR and HSQC spectra disclosed 22 resonances, which were attributed to two methyls at $\delta_{\mathrm{C}} 10.9(\mathrm{C}-1)$ and $22.1(\mathrm{C}-20)$, two oxymethines at $\delta_{\mathrm{C}} 72.3(\mathrm{C}-3)$ and $68.3(\mathrm{C}-19)$, two azamethines at $\delta_{\mathrm{C}} 55.6(\mathrm{C}-10)$ and $54.9(\mathrm{C}-13)$, two olefinic methines at $\delta_{\mathrm{C}} 134.5(\mathrm{C}-4)$ and $131.6(\mathrm{C}-5)$ within the seven membered ring, giving the sixth degree of unsaturation, one methine at $\delta_{\mathrm{C}} 54.1(\mathrm{C}-14)$, and nine methylene groups at $\delta_{\mathrm{C}} 30.4$ (C-2), 24.6 (C-6), 38.4 (7), 38.7 (C-9), 31.0 (C-11), 31.0 (C-12), 33.6 (C-16), 19.7 (C-17), and 32.9 (C-18). Four quaternary resonances, including two $\mathrm{sp}^{3}$ spiro-carbons at $\delta_{\mathrm{C}} 85.1(\mathrm{C}-8)$ and $82.8(\mathrm{C}-15)$ and two downfield resonances at $\delta_{\mathrm{C}} 150.8(\mathrm{C}-21)$ and $181.6(\mathrm{C}-22)$, are attributed to the guanidinic and free carboxylic acid functionalities, giving the remaining two degrees of unsaturation and satisfying the eight degrees required by HR-MS. The $2 \mathrm{D}$ NMR spectral data interpretations, including ${ }^{1} \mathrm{H}-{ }^{1} \mathrm{H}$ COSY, and ${ }^{1} \mathrm{H}-{ }^{13} \mathrm{C}$ HMBC, enabled us to establish and confirm the total structure of 1 and supported its identification as crambescidin acid, a pentacyclic guanidine alkaloid previously isolated from another species, Monanchora unigiculata [22]; however, it is recorded here for the first time from Monanchora $\mathrm{n}$. sp. Compound $\mathbf{1}$ displayed promising cytotoxicity against Kb cell lines with inhibitory activities of $(100 \pm 1)$ and $(80 \pm 1)$ at concentrations of 10 and $1 \mu \mathrm{M}$, respectively.

1) Institut de Chimie des Substances Naturelles, CNRS UPR 2301, Univ. Paris-Sud, University of Paris-Saclay, 1, Avenue de la Terrasse, 91198 Gif-sur-Yvette, France, e-mail: eldemerdash555@gmail.com; 2) Organic Chemistry Division, Chemistry Department, Faculty of Science, Mansoura University, 35516, Mansoura, Egypt; 3) Institut de Recherche Pour le Developpement, Unite Mixte de Recherche 241, Ecosystemes Insulaires Oceaniens, BP529, 98713, Papeete, Tahiti, French Polynesia; 4) Institut de Recherche Pour le Developpement, Unite Mixte de Recherche 6539, Laboratoire des Sciences de L'environnement Marin, rue Dumont d'Urville, 29280, Plouzane, France. Published in Khimiya Prirodnykh Soedinenii, No. 6, November-December, 2020, pp. 1007-1008. Original article submitted October 21, 2019. 


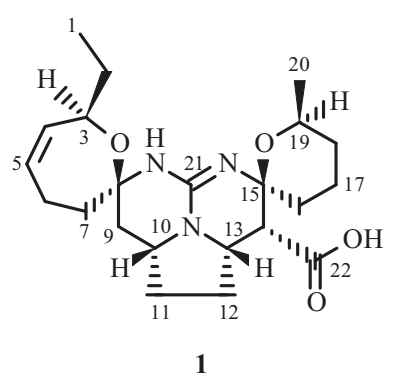

The co-isolated simple nitrogenous compounds $\mathbf{2}-\mathbf{5}$ were identified by matching their ${ }^{1} \mathrm{H}$ NMR and HR-MS spectral data with the literature $[23,24]$.

General experimental procedures, extraction, isolation, and cytotoxic activity, were performed according to previously published protocol [7].

Animal Material. The sponge was collected off the coast of Hiva Oa $\left(9^{\circ} 45.421^{\prime} \mathrm{S}-139^{\circ} 08.275^{\prime} \mathrm{W}\right)$ at $20 \mathrm{~m}$ depth using Scuba on 07.09.2009. It was identified as Monanchora n. sp. (OTU QM4696) and a reference specimen was deposited at the Queensland Museum (Brisbane, Australia) under accession number QM G331116 [25].

Crambescidin acid (1), pale yellow oil (11.5 mg). HR-ESI-MS m/z 404.2461 [M + H] ${ }^{+}$(calcd for $\mathrm{C}_{22} \mathrm{H}_{34} \mathrm{~N}_{3} \mathrm{O}_{2}$, 404.2549). UV (MeOH, $\left.\lambda_{\max }, \mathrm{nm}\right)(\log \varepsilon): 276.4$ (0.05). IR (neat, $v_{\max }, \mathrm{cm}^{-1}$ ): 3230, 2967, 2932, 1630, 1542, 1448, 1343, 1114, 1054, 1010, 965, 844, 754. ${ }^{1} \mathrm{H}$ NMR (500 MHz, CD $\mathrm{OD}, \delta$, ppm, J/Hz): $5.71(1 \mathrm{H}, \mathrm{m}, \mathrm{H}-5), 5.51(1 \mathrm{H}, \mathrm{dd}, \mathrm{J}=11,2.0$, $\mathrm{H}-4), 4.35$ (1H, m, H-3), 4.23 (1H, m, H-13), 4.03 (1H, m, H-10), 3.82 (1H, m, H-19), 2.69 (1H, d, J = 5.0, H-14), $2.59(1 \mathrm{H}$, dd, J = 5.0, 4.5, H-9a), 2.42 (1H, m, H-7a), 2.40 (1H, m, H-6a), 2.32 (4H, m, H-11, 12), 2.13 (1H, m, H-6b), 1.95 (1H, dd, J = 6.5, 6.0, H-7b), 1.76 (2H, m, H-17), $1.71(2 \mathrm{H}, \mathrm{m}, \mathrm{H}-16), 1.68(2 \mathrm{H}, \mathrm{m}, \mathrm{H}-18), 1.54(1 \mathrm{H}, \mathrm{m}, \mathrm{H}-2 \mathrm{a}), 1.46(1 \mathrm{H}, \mathrm{m}, \mathrm{H}-2 \mathrm{~b}), 1.25(1 \mathrm{H}$,

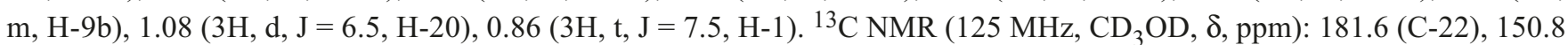
(C-21), 134.5 (C-4), 131.6 (C-5), 85.1 (C-8), 82.8 (C-15), 72.3 (C-3), 68.3 (C-19), 55.6 (C-10), 54.9 (C-13), 54.1 (C-14), 38.7 (C-9), 38.4 (C-7), 33.6 (C-16), 32.9 (C-18), 31.0 (C-11, 12), 30.4 (C-2), 24.6 (C-6), 22.1 (C-20), 19.7 (C-17), 10.9 (C-1).

\section{ACKNOWLEDGMENT}

Financial support from CNRS-ICSN, IRD for the collecting trip aboard R/V Alis, French and French Polynesian Government for the Marquesas Project, as well as ANR (POMARE Project, 2011-EBIM-006-01) are gratefully acknowledged. Amr El-Demerdash's Ph.D. was funded, and completely financed, by a grant from the Egyptian Government (Egyptian cultural Bureau in Paris), which is gratefully acknowledged. We thank F. Pelissier and O. Thoison for HPLC assistance. We give special thanks to J. F. Gallard and K. Hammad for technical assistance and NMR measurements, and to T. Cresteil and G. Aubert for cytotoxicity evaluations.

\section{REFERENCES}

1. J. Carlos, ACS Med. Chem. Lett., 9, 959 (2018).

2. A. R. Carroll, B. R. Copp, R. A. Davis, R. A. Keyzers, and M. R. Prinsep, Nat. Prod. Rep., 36, 122 (2019).

3. D. Sipkema, M. C. R. Franssen, R. Osinga, R. J. Tramper, and R. H. Wijffels, Mar. Biotechnol., 7, 142 (2005).

4. $\quad$ M. F. Mehbub, J. Lei, C. Franco, and W. Zhang, Mar. Drugs, 12, 4539 (2014).

5. S. A. M. Khalifa, N. Elias, M. A. Farag, L. Chen, A. Saeed, M. E. F. Hegazy, M. S. Moustafa, A. Abd El-Wahed, S. M. Al-Mousawi, S. G. Musharraf, F. R. Chang, A. Iwasaki, K. Suenaga, M. Alajlani, U. Goransson, and H. R. El-Seedi, Mar. Drugs, 17, 491 (2019).

6. A. El-Demerdash, A. G. Atanasov, A. Bishayee, M. Abdel-Mogib, J. N. A. Hooper, and A. Al-Mourabit, Nutrients, 10, 33 (2018).

7. A. El-Demerdash, C. Moriou, M. T. Martin, A. Rodrigues-Stien, S. Petek, M. Demoy-Schnider, K. Hall, J. N. A Hooper, C. Debitus, and A. Al-Mourabit, J. Nat. Prod., 79, 1929 (2016).

8. P. E. Campos, E. F. Queiroz, L. Marcourt, J. L. Wolfender, A. S. Sanchez, B. Illien, A. Al Mourabit, and A. Gauvin-Bialecki, Planta Med., 82, S1 (2016). 
9. A. El-Demerdash, C. Moriou, M. T. Martin, S. Petek, C. Debitus, and A. Al-Mourabit, Nat. Prod. Res., 32, 1512 (2018).

10. I. I. Kapustina, K. M. Tabakmakher, and T. N. Makar'eva, Chem. Nat. Compd., 47, 1025 (2012).

11. W. Wang, B. Mun, Y. Lee, M. V. Reddy, Y. Park, J. Lee, H. Kim, D. Hahn, J. Chin, M. Ekins, and S. J. Nam, J. Nat. Prod., 76, 170 (2013).

12. W. Wang, T. G. Lee, R. S. Patil, B. Mun, I. Yang, H. Kim, D. Hahn, D. H. Won, J. Lee, Y. Lee, and H. Choi, J. Nat. Prod., 78, 368 (2015).

13. A. El-Demerdash, S. Petek, C. Debitus, and A. Al-Mourabit, Chem. Nat. Compd., 54, 1143 (2018).

14. P. E. Campos, J. M. Wolfender, E. F. Queiroz, L. Marcourt, A. Al-Mourabit, M. Frederich, A. Bordignon, N. De Voogd, B. Illien, and A. Gauvin-Bialecki, J. Nat. Prod., 80, 1404 (2017).

15. K. M. Tabakmakher, T. N. Makarieva, L. K. Shubina, V. A. Denisenko, R. S. Popov, A. S. Kuzmich, H. S. Lee, and V. A. Stonik, Nat. Prod. Commun., 11, 1817 (2016).

16. S. Takishima, A. Ishiyama, M. Iwatsuki, K. Otoguro, H. Yamada, S. Omura, K. Kobayashi, R. W. M. van Soest, and S. Matsunaga, Org. Lett., 11, 2655 (2009).

17. K. A. El-Sayed, Stud. Nat. Prod. Chem., 24, 473 (2000).

18. A. El-Demerdash, L. Ermolenko, E. Gros, P. Retailleau, B. N. Thanh, G. B. Anne, and A. Al-Mourabit, Eur. J. Org. Chem., 2020, 5677 (2020).

19. A. El-Demerdash, C. Moriou, J. Toullec, M. Besson, S. Soulet, N. Schmitt, S. Petek, D. Lecchini, C. Debitus, and A. Al-Mourabit, Mar. Drugs, 16, 146 (2018).

20. A. El-Demerdash, M. A. Tammam, A. G. Atanasov, J. N. A. Hooper, A. Al-Mourabit, and A. Kijjoa, Mar. Drugs, 16, 214 (2018).

21. A. El-Demerdash, A. G. Atanasov, O. K. Horbanczuk, M. A. Tammam, M. Abdel-Mogib, J. N. A. Hooper, N. Sekeroglu, A. Al-Mourabit, and A. Kijjoa, Mar. Drugs, 17, 115 (2019).

22. K. M. Meragelman, T. C. McKee, and J. B. McMahon, J. Nat. Prod., 67, 1165 (2004).

23. E. Regalado, J. Mendiola, A. Laguna, C. Nogueiras, and O. P. Thomas, Nat. Prod. Commun., 5, 1187 (2010).

24. Spectral Data for Organic Compounds, http://sdbs.db.aist.go.jp/sdbs/cgi-bin/direct_frame_top.cgi.

25. C. Debitus, BSMPF-1 cruise, Alis R/V, http://dx.doi.org/10.17600/9100030 (2009). 